\title{
Study on Particles Wetted by the Calcium-Based Slurries and the Slurries Viscosity with the Wetting Agents
}

\author{
Xiaowen Hao ${ }^{1, *}$, Zhijun Zhang ${ }^{1}$ and Ran Liu $^{2}$ \\ ${ }^{I}$ School of Automotive Engineering, Harbin Institute of Technology at Weihai, Weihai, China; ${ }^{2}$ Harbin Institute of Tech- \\ nology (Weihai) Library, Harbin Institute of Technology at Weihai, Weihai, China
}

\begin{abstract}
Chemical agglomeration can reduce the particulates (including PM2.5) concentration emitted from fly ash particulates and the wet flue gas desulfurization (WFGD) system. However, the calcium-based slurries affect the regulation of agglomeration compared to water, in particular, the wettability of the particulates and the viscosity of the calciumbased slurry. The wettability and the viscosity are the important factors affecting agglomeration, conversely. This manuscript studied the two factors affecting the calcium-based slurries. The sessile drop method was used to measure the contact angle. Four kinds of calcium-based slurries $(\mathrm{CaCO} 3, \mathrm{CaSO} 3, \mathrm{CaSO} 4, \mathrm{Ca}(\mathrm{OH}) 2)$ respectively were mixed with five kinds of wetting agents' wet particles, and the wetting regulation was studied. The viscometer was used to measure the viscosity of the calcium-based slurry with the wetting agents, and the variation of the viscosity was analyzed as well. The results showed that the smaller the particle size was, the more it is difficult to be wet, and the wetting agents could enhance the wettability greatly, but the effect was related to the properties of the calcium-based material and the wetting agents. There were variable viscosities of different calcium-based slurries after adding the wetting agents. Overall, the Compound No. 3 had the optimal qualities in improving particle wettability and maintaining the calcium-based slurry with a high viscosity. Therefore, the Compound No. 3 is considered the optimal in facilitating chemical agglomeration.
\end{abstract}

Keywords: Calcium-based slurry, contact angle, particles, viscosity.

\section{INTRODUCTION}

PM2.5 from coal-fired power plants is one of the important sources in the Chinese atmosphere [1-2]. The Chinese government has implemented the most strict emission standard of the world with regards to the air pollutants for the power plants since 2012, which is called as close to zero emission. The Chinese government has been encouraging these plants to satisfy the gas turbines emission standard since 2014. A substantial reward has been given by the Chinese government to those plants that satisfied gas turbines emission standard, since 2015. However, the existing dust removal devices are very difficult to meet this very strict standard, let alone the PM2.5 concentration which is more likely to increase after the wet flue gas desulfurization (WFGD) system [3]. The wet electrostatic precipitators (WESP) can not only further collect PM2.5, but also it requires much investment and land occupation. And it also causes corrosion problems as well. Furthermore, the number of concentration of PM2.5 is still up to several hundred thousand per cubic centimeter even though the emission standard is satisfied [4], which is harmful to human health.

Chemical agglomeration refers that particles wet by agglomeration solution bond and grow by the adhesion effect, which can effectively reduce the mass and the concentration of PM2.5.

*Address correspondence to this author at the School of Automotive Engineering, Harbin Institute of Technology at Weihai, 264209, Weihai, China; Tel: +86-631-5687862; Fax: +86-631-5687210; E-mail: haoxiaowen@sohu.com
Liu [5] found that the chemical agglomeration can reunite PM2.5 four times higher than the average particle size, reducing the concentration of PM2.5 by more than $40 \%$, and the removal efficiency of electrostatic precipitators for PM2.5 can be increased by approximately $40 \%$, while that of WFGD system is $30 \%$ relatively. However, the applications of chemical agglomeration were slowly developed due to the expensive agglomeration solution in recent years.

Agglomeration solution conventionally includes high polymer binders, wetting agent and water. The role of wetting agents is to improve the particles wetting property [6-7]. To some degree, the agglomeration ability of calcium-based material in desulfurization slurry was like high polymer binders [8], the reason is that the calcium-based material has adhesive. Geng [9] found that the slurry could improve the PM2.5 agglomeration effect in WFGD with the wetting agents. However, the calcium-based material included Ca$\mathrm{CO}_{3}, \mathrm{CaSO}_{3}, \mathrm{CaSO}_{4}$, or $\mathrm{Ca}(\mathrm{OH})_{2}$. The agglomerating and wetting particles ability for each calcium-based material has not been researched till now.

The greater the concentration of adhesives is, the stronger the adhesion capacity reflected in viscosity will be, and the faster the particles will grow during the study of chemical agglomeration. Agglomeration rate of the particles and viscosity of the solution are almost proportional [10-12]. Dong [13] found that the calcium-based materials in WFGD system had certain abilities of agglomeration due to their viscosity. But its agglomeration is unconscious action. 
The calcium-based slurry with wetting agents shows the agglomeration ability in the WFGD system, so it will be possible to achieve the agglomeration of PM2.5 without using expensive polymer binders to a certain extent. In this paper, the regulation of each calcium-based materials (e.g. $\mathrm{CaCO}_{3}$, $\mathrm{CaSO}_{3}, \mathrm{CaSO}_{4}$, and $\mathrm{Ca}(\mathrm{OH})_{2}$ ) slurries with wetting agents wets particles, and the regulation of slurry viscosity is studied based on experiments.

\section{EXPERIMENTAL DEVICE AND METHOD}

\subsection{Experimental Samples and Reagents}

The fly ash particles used in this experiment came from a thermal power plant in Weihai, China, which were collected after ESP. The particle size range in the experiments was:

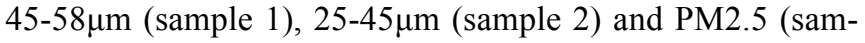
ple 3 ).

The cationic surfactants are not suitable for the wetting agents [14]. And in this study, the wetting agents were: Triton X-100 and Tween 80, which were non-ionic wetting agents; Sodium dodecyl benzene sulfonate (SDBS), sodium dodecyl sulfate (SDS) and Compound No. 3, which were anionic wetting agents.

All wetting agents were dissolved in the calcium-based slurry, and the mass concentration of the agents was $0.025 \%$. The concentration of $\mathrm{CaCO}_{3}, \mathrm{CaSO}_{3}, \mathrm{Ca}(\mathrm{OH})_{2}$ slurries was $10 \%, 15 \%, 20 \%, 25 \%$ and $30 \%$. The concentration of $\mathrm{CaSO}_{4}$ slurry was $10 \%, 12.5 \%, 15 \%, 17.5 \%$ and $20 \%$, since $\mathrm{CaSO}_{4}$ slurry turned into solid very quickly at high concentrations.

\subsection{Experimental System and Method}

\subsubsection{Wetting Experiments}

Wettability of fly ash particles is usually measured via the contact angle. There are a few methods to measure the contact angle, but only sessile drop method can be used in the experiments because of the existence of a large number of particles in the slurry. The images of the calcium-based slurry wetting particles were recorded by high-speed camera MEMRECAM GX-3, as shown in Fig. (1). The contact angle hypsometry was analyzed by a computer.

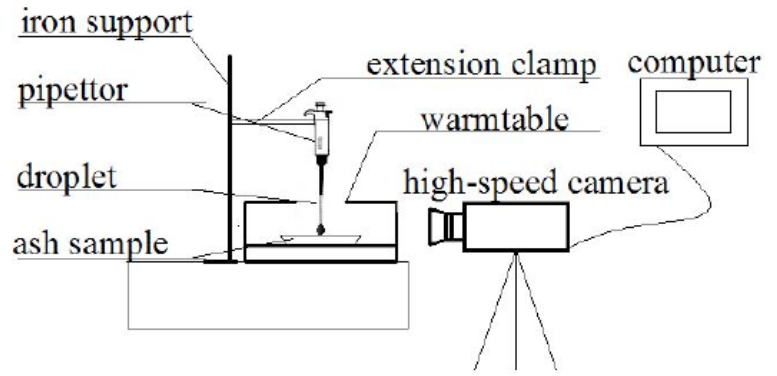

Fig. (1). Schematic diagram of the contact angle measuring system.

Experimental procedure was as follows. The $3.8 \mathrm{~g}$ fly ash particles were uniformly loaded in the crucible whose volume was $3 \mathrm{ml}$, and the exit surface of the crucible needed to remain smooth, ensuring that each experimental ash in the crucible got the same bulk density. Additionally, the configured calcium-based slurry was heated to $38{ }^{\circ} \mathrm{C}$ in the water bath and stirred for at least 3 minutes, then the liquid drops were quickly titrated on the particles surface with pipettes when the temperature dropped to $30^{\circ} \mathrm{C}$ accurately. Meanwhile, the high-speed camera recorded the process of droplet wetting the particles, and the temperature of the particles sample was kept at $30^{\circ} \mathrm{C}$ over the whole process.

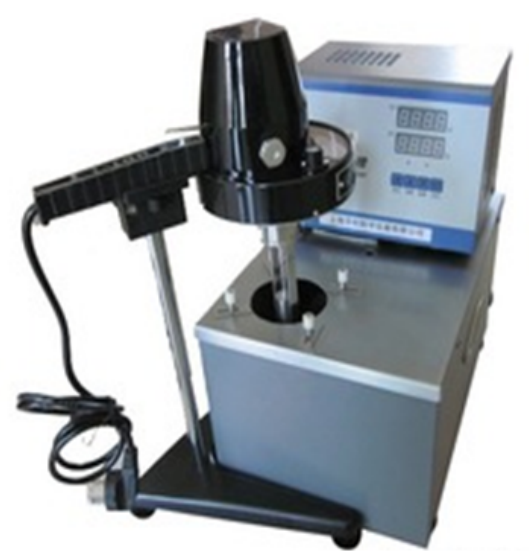

Fig. (2). Rotational viscometer and thermostatic water bath.

\subsubsection{The Experiment of Viscosity}

Fig. (2) shows a rotational viscometer NDJ-1. It was placed in a water bath device $\mathrm{CH} 1006$, whose measurement range was $0 \sim 100 \mathrm{mPa} \cdot \mathrm{s}$. It can measure the viscosity of the calcium-based slurry. The viscosity was recorded when the viscometer dial was stable on-stream.

\section{EXPERIMENTAL RESULTS AND ANALYSIS}

\subsection{Experimental Study of the Calcium-Based Slurry Wetting Particles}

\subsubsection{Effect of Wetting Agents on the Contact Angle of the Calcium-Based Slurry}

The sample 1 was tested in this part of the experiment.

Fig. (3a) presents the characteristic curve of the contact angle of $\mathrm{CaCO}_{3}$ slurry mixed with wetting agents wetting particles. The wetting agents can improve the wettability of the particles significantly. The order of wettability under the same slurry concentration from good to poor is: $\mathrm{CaCO}_{3}$ slurry with SDBS, SDS, Compound No. 3, Tween 80, Triton X10 , and the pure slurry in the last. The cloud point of nonionic wetting agents will decline sharply in weakly alkaline $\mathrm{CaCO}_{3}$ slurry, resulting in the hydrophobicity and the wettability of $\mathrm{CaCO}_{3}$ slurry which may have been deteriorated or lowering [14]. Overall, the $\mathrm{CaCO}_{3}$ slurry-added anionic wetting agents have better effectiveness.

Fig. (3b) presents the characteristic curve of the contact angle of $\mathrm{CaSO}_{3}$ slurry with the wetting agents wetting particles. The order of wettability under the same slurry concentration from good to poor is: $\mathrm{CaSO}_{3}$ slurry with Compound No. 3, Triton X-100, Tween 80, SDBS, SDS, and the pure slurry in the last. The surface active ions of the anionic wetting agents repulse each other when the ions are adsorbed on the surface or aggregate into micelles. Furthermore, the less close the structure arrangement of hydrocarbon chain is, the greater the surface tension of suspension will be [15]. 


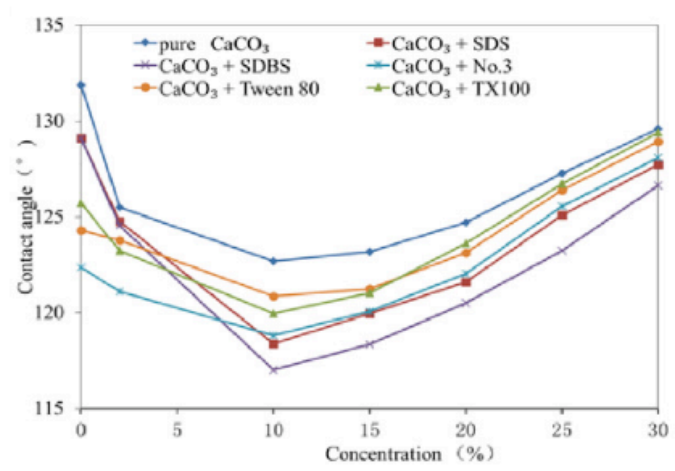

(a) $\mathrm{CaCO}_{3}$

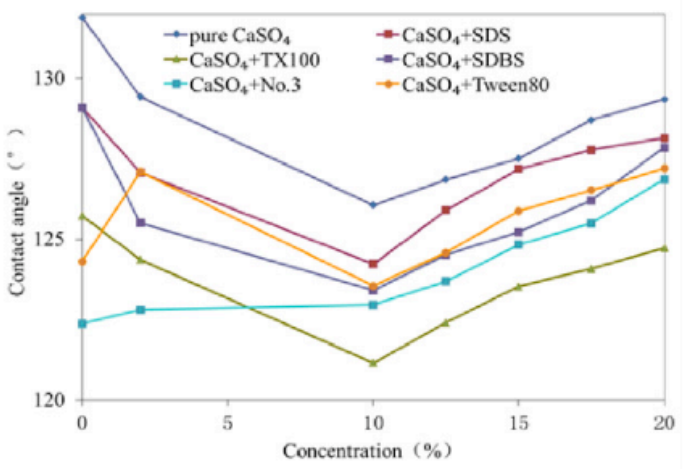

(c) $\mathrm{CaSO}_{4}$

Fig. (3). Contact angle of the calcium-based slurry wetting particles.

Thus, the wetting effect on the particles of the anionic wetting agents is fairly worse than the non-ionic wetting agent. However, the Compound No. 3 displays the quite different wetting characteristic compared to the usual anionic wetting agents, which is thus the best wetting agent for the $\mathrm{CaSO}_{3}$ slurry.

Fig. (3c) presents the characteristic curve of the contact angle of $\mathrm{CaSO}_{4}$ slurry with wetting agents wetting particles. The order of wettability under the same slurry concentration from good to poor is: $\mathrm{CaSO}_{4}$ slurry with Triton X-100, Tween 80, Compound No. 3, SDBS, SDS, and the pure $\mathrm{CaSO}_{4}$ slurry in the last. It could be inferred from the above that sulfate could decompose rapidly under self-catalysis because $\mathrm{CaSO}_{4}$ slurry has weak acidity. In contrast, the nonionic wetting agents do not decompose and have stable properties, and they tend to form micelles more easily. So the non-ionic wetting agents dissolved in the $\mathrm{CaSO}_{4}$ slurry have better wetting effect than the anionic wetting agents.

Fig. (3d) presents the characteristic curve of the contact angle of $\mathrm{Ca}(\mathrm{OH})_{2}$ slurry with wetting agents wetting particles. The order of wettability under the same slurry concentration from good to poor is: $\mathrm{Ca}(\mathrm{OH})_{2}$ slurry mixed with Compound No. 3, Tween 80, Triton X-100, SDBS, SDS, and the pure $\mathrm{Ca}(\mathrm{OH})_{2}$ slurry in the last. Charges in $\mathrm{Ca}(\mathrm{OH})_{2}$ slurry with non-ionic wetting agents move to the direction of positive charge, reducing the electrostatic repulsion, and thereby making it easier to wet the particles surface. The slurry with general anionic wetting agents, by comparison, presents the opposite effect. However, the Compound No. 3

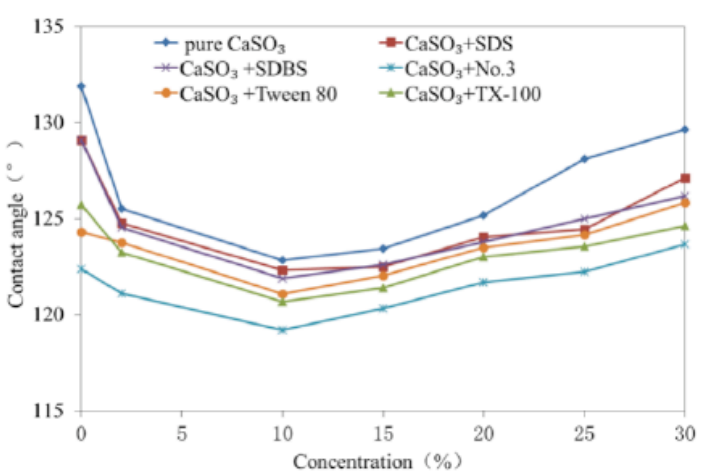

(b) $\mathrm{CaSO}_{3}$

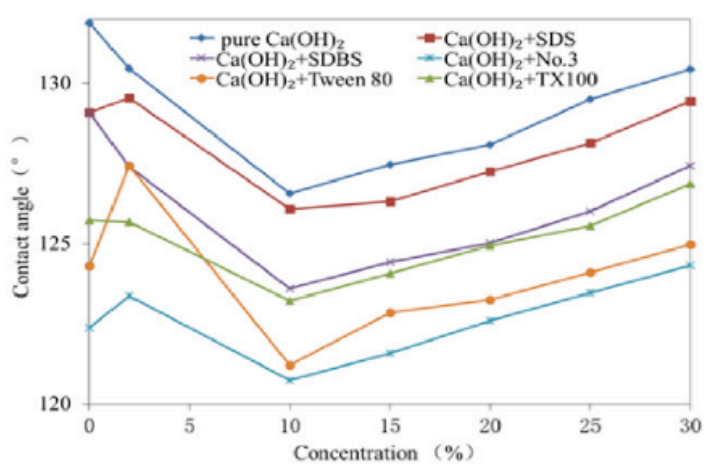

(d) $\mathrm{Ca}(\mathrm{OH})_{2}$

displays the qualities different from the general anionic wetting agents, and it still be the best wetting agent for $\mathrm{Ca}(\mathrm{OH})_{2}$ slurry.

In summary, the calcium-based slurries with wetting agents can significantly reduce the contact angle of those wetting particles. Various calcium-based slurries obtain the best wettability when the slurries concentration is about $10 \%$. The contact angle is still greater than $90^{\circ}$, that is to say, the fly ash particles belong to the category of difficult to be wetted. Furthermore, the wettability mainly relates to the $\mathrm{pH}$ of the slurries, the formation of micelle and the charge distribution, and $\mathrm{pH}$ is the most important factor among the three. All analyses reveal that anionic surfactants are better for $\mathrm{CaCO}_{3}$ slurry; non-ionic surfactants are better for others. What should be emphasized is that the Compound No. 3 could improve the wettability of all the slurries significantly.

\subsubsection{Effect of Particle Size on the Contact Angle}

This section is devoted to the relevant research of contact angle related to different particles size. Considering the wetting properties of the wetting agents, the Compound No. 3 had the best comprehensive wetting properties among all of the four calcium-based slurries; concentration of the calcium-based slurries was $10 \%$, while that of the Compound No. 3 was $0.025 \%$. Particle size has a great influence on the contact angle as shown in Fig. (4). The gaps between the larger particles are larger than that of the smaller ones during the accumulation process. The larger gaps cause the larger equivalent capillary radius [16]. So the wetting rate and the 


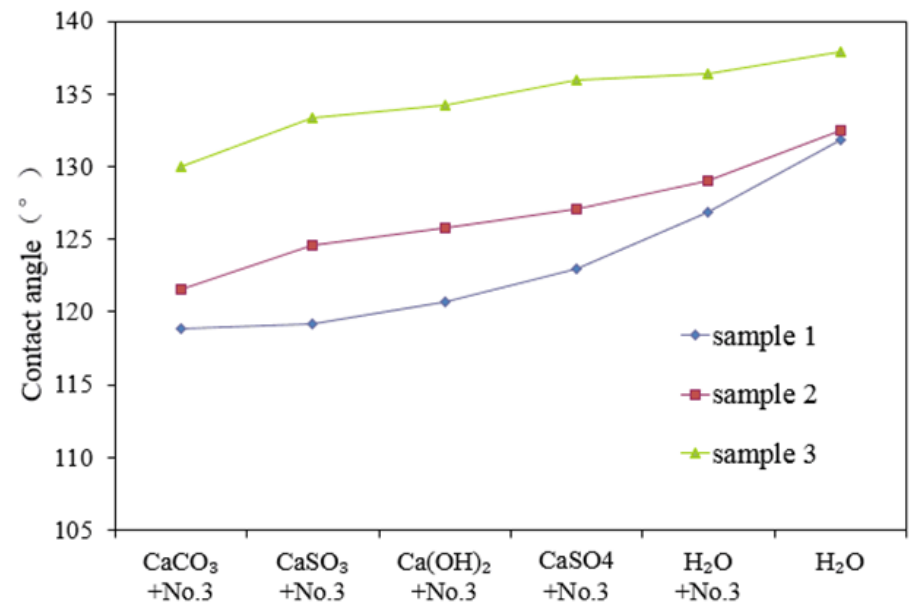

Fig. (4). Effect of the particles size on the contact angle.

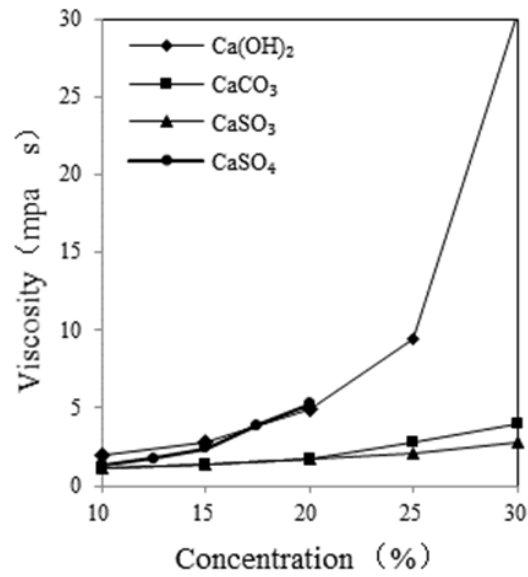

Fig. (5). Effect of the concentration on viscosity.

wetting ability of the large particles are better than that of the small one. In contrast, fine particle surfaces could form gas films easily, which lead to its bad wettability, too.

\subsection{Viscosity of Calcium-Based Slurry}

\subsubsection{Effect of Concentration on the Viscosity}

The viscosity of deionized water is $1.1 \mathrm{mPa} \bullet \mathrm{s}$ from the experiment. The characteristic curve of viscosity at different calcium-based slurries concentration is shown in Fig. (5). The force between the solid-material contained in the slurry increases with the concentration, which results in the rise of the slurry viscosity. From the point of view of chemical agglomeration, the $\mathrm{Ca}(\mathrm{OH})_{2}$ and the $\mathrm{CaSO}_{4}$ slurries have much larger viscosity and have larger agglomeration ability than that of the $\mathrm{CaCO}_{3}$ and the $\mathrm{CaSO}_{3}$ slurries.

\subsubsection{Effect of Wetting Agents on the Viscosity}

The characteristic curves of the viscosity of the calciumbased slurry with the wetting agents are shown in Fig. (6). Viscosity of the $\mathrm{CaCO}_{3}$ or the $\mathrm{CaSO}_{3}$ slurry increases with the concentration of the corresponding calcium-based slurry. A few wetting agents can increase the slurry viscosity significantly, and the Compound No. 3 has the highest effect on the five wetting agents. In addition, TX-100 only reduces the viscosity of the $\mathrm{Ca}(\mathrm{OH})_{2}$ slurry, while the addition of the other four wetting agents increases slurry viscosity to different degrees. But all of the wetting agents reduce the viscosity of the $\mathrm{CaSO}_{4}$ slurry, and the difference between the pure slurry and the slurry with those wetting agents increases with the concentration. Increase in the calcium-based slurry viscosity in order to improve PM2.5 agglomeration needs be selected based on the calcium-based material and the wetting agents.

\section{ANALYSIS AND CONCLUSION}

The smaller the particle size is, the more difficult it is to be wetted, thus the agglomeration of PM2.5 is harder than the larger particles. The wettability of the fly ash particles could be improved via the calcium-based slurries with the wetting agents. The Compound No. 3 is the optimal wetting agent among the five agents in the calcium-based slurries used in the study.

Through the comparison of the four kinds of the calciumbased slurries, it can be found that the compound No. 3 not only significantly increased viscosity of $\mathrm{CaCO}_{3}, \mathrm{CaSO}_{3}$, and $\mathrm{Ca}(\mathrm{OH})_{2}$ slurries, but also decreased the viscosity of $\mathrm{CaSO}_{4}$ slurry at the minimum. The Compound No. 3 is the optimal agglomeration promoter, thereby increasing the slurry adhesiveness. 


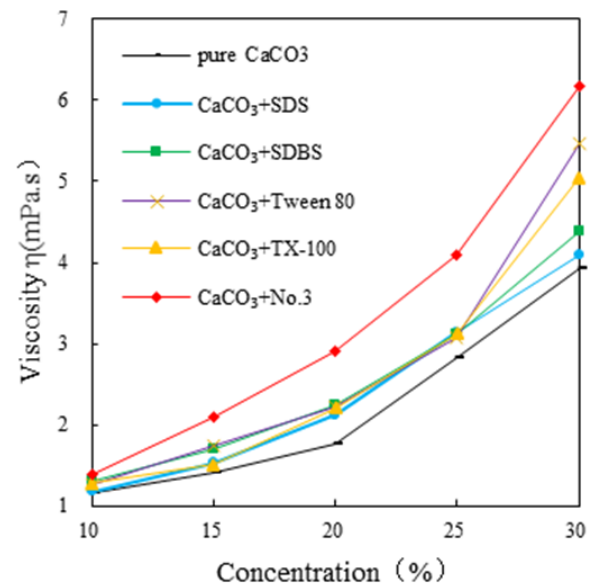

(a) $\mathrm{CaCO}_{3}$

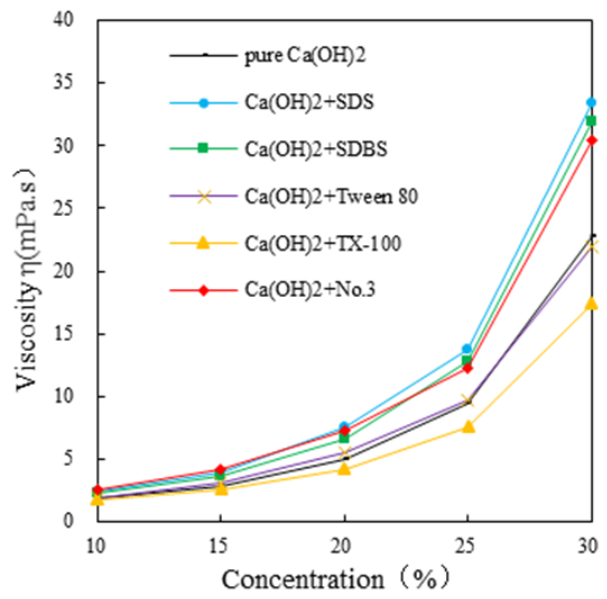

(c) $\mathrm{Ca}(\mathrm{OH})_{2}$

Fig. (6). Viscosity of the calcium-based slurries with the wetting agents.

In summary, the Compound No. 3 showed the best ability to improve the wettability, adhesiveness and the PM2.5 agglomeration when the chemical agglomeration is applied in the WFGD system.

\section{CONFLICT OF INTEREST}

The authors confirm that this article content has no conflict of interest.

\section{ACKNOWLEDGEMENTS}

This work is supported by the Natural Science Foundation of Shandong Province (Grant No. ZR2013EEM001) and the Science and Technology Development Project of Shandong Province (Grant No. 2014GSF117007).

\section{REFERENCES}

[1] R. Zhang, J. Jing, J. Tao, S.C. Hsu, G. Wang, J. Gao, C.S.L. Lee, and L. Zhu, "Chemical Characterization and Source Apportionment of PM 2.5 in Beijing: Seasonal Perspective," Atmospheric Chemistry and Physics, vol. 13, no. 14, pp. 7053-7074, 2013.

[2] X.M. Gao, L.X. Yang, S.H. Cheng, R. Gao, Y. Zhou, and L.K. Xue, "Semi-continuous Measurement of Water-soluble Ions in PM 2.5 in Jinan, China: Temporal Variations and Source Apportionments," Atmospheric Environment, vol. 45, no. 33, pp. 6048-6056, 2011.

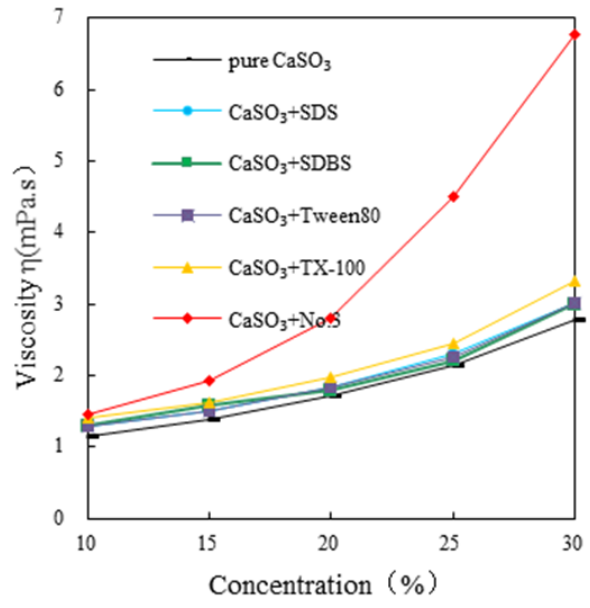

(b) $\mathrm{CaSO}_{3}$

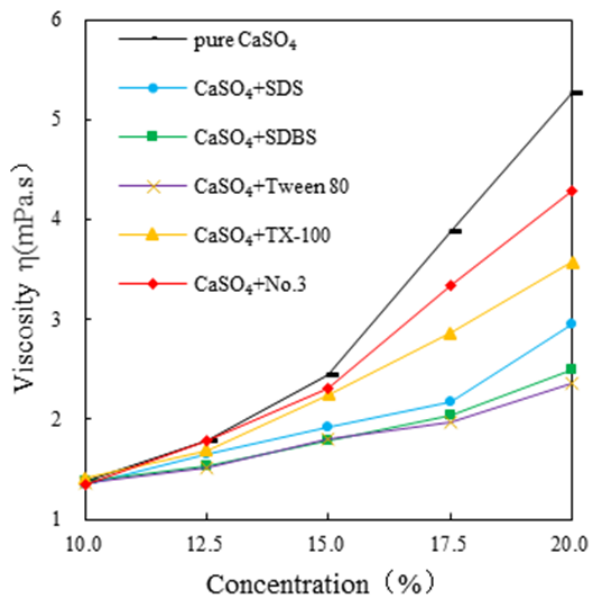

(d) $\mathrm{CaSO}_{4}$
[3] W. Zhao, Y. Liu, J. Bao, J. Geng, and L. Yang, "Experimental Research on Fine Particles Removal from Flue Gas by Chemical Agglomeration," Proceedings of the CSEE, vol. 33, no. 20, pp. 5258,2013

[4] Z. Li, "Research on PM2.5 Production and Emission Characteristics of Thermal Power Plant Pulverized Coal Boiler," M.S. Thesis, Harbin Institute of Technology, 2013.

[5] Y. Liu, L.J. Yang, and W. Zhao, "Pollutant Control Equipment in Coal-fired Power Station Boosts up Methods of PM 2.5 Removal," China Environmental Protection Industry, no. 3, pp. 43-46, 2013.

[6] J.P. Yan, L.J. Yang, and X.L. Shen, "Wettability of PM2.5 from Combustion," Journal of Southeast University (Natural Science Edition), vol. 36, no. 5, pp. 760-764, 2006.

[7] H.L. Li, J.Y. Zhang, Y.C. Zhao, C.Y. Wu, C.G. Zheng, "Wet ability of Fly Ashes from Four Coal-Fired Power Plants in China," Industrial \& Engineering Chemistry Research, vol. 50, pp. 77637771, 2011.

[8] J.H. Gao, J.X. Liu, J.M. Gao, "Modelling and Experimental Study on Agglomeration of Particles from Coal Combustion in Multistage Spouted Fluidized Tower," Advanced Powder Technology, vol. 20, pp. 375-382, 2009

[9] J.F. Geng, S.J. Song, J.J. Bao, and L.J. Yang, "Improving Performance of WFGD System to Remove Fine Particles by Adding Wetting Agents," CIESC Journal, vol. 62, no. 4, pp. 1084-1090, 2011.

[10] P. Rajniak, C. Mancinellia, R.T. Cherna, F. Stepanekb, L. Farbera, and B.T. Hill "Experimental Study of Wet Granulation in Fluidized Bed: Impact of the Binder Properties on the Granule Morphology," International Journal of Pharmaceutics, vol. 334, pp. 92-102, 2007. 
[11] D. O. James, "Binder Addition Methods and Binder Distribution in High Shear and Fluidised Bed Granulation," Chemical Engineering Research and Design, vol. 89, no. 5, pp. 553-559, 2011.

[12] F. Wei, "Study on Sub-micron Particle Formation and Agglomeration Mechanism from Coal Combustion," Huazhong University of Science and Technology, 2005.

[13] Y. Dong, G.J. Qi, L. Cui, and C.Y. Ma, "Analysis on Agglomeration of Humidified Particles in CFB-FGD Process," Journal of Power Engineering, vol. 29, no. 7, pp. 671-675, 2009.
[14] X. R. Teng, "Surface Physical Chemistry," Chemical Industry Press, 2009.

[15] S. Heidenreich, "Condensational Droplet Growth as a Preconditioning Technique for the Separation of Submicron Particles From Gases," Chemical Engineering and Processing: Process Intensification, vol. 34, no. 3, pp. 235-244, 1995.

[16] A. Tahani, H. Van Damme, C. Noik, and P. Levitz, "Adsorption of Non-Ionic surfactants on Kaolins," Journal of Colloid and Interface Science, vol. 184, no. 2, pp. 469-476, 1996.

Received: May 26, 2015

Revised: July 14, 2015

Accepted: August 10, 2015

(C) Hao et al.; Licensee Bentham Open.

This is an open access article licensed under the terms of the (https://creativecommons.org/licenses/by/4.0/legalcode), which permits unrestricted, noncommercial use, distribution and reproduction in any medium, provided the work is properly cited. 